\title{
Case - Resolution of urethral mesh perforation after sling: The migrating mesh
}

Sara Luisa Jones, $\mathrm{BHSc}^{1}$; Yanbo Guo, $\mathrm{MD}^{2}$; Laura Nguyen, $\mathrm{MD}^{2}$

${ }^{1}$ Dalhousie Medical School, Dalhousie University, Halifax, NS, Canada; ${ }^{2}$ Division of Urology, Department of Surgery, McMaster University, Hamilton, ON, Canada

Cite as: Jones SL, Guo Y, Nguyen L. Case - Resolution of urethral mesh perforation after sling: The migrating mesh. Can Urol Assoc J 2020 August 7; Epub ahead of print. http://dx.doi.org/10.5489/cuaj.6645

Published online August 7, 2020

$* * *$

\section{Introduction}

Stress urinary incontinence (SUI) is a common issue that affects up to $50 \%$ of women. ${ }^{1} \mathrm{Mid}-$ urethral slings are an effective surgical intervention with short and long term success rates near $90 \% .^{2}$ Although complications are relatively low, risks remain, including mesh complications, infection, pain, urinary obstruction, and de novo urgency., ${ }^{3,4}$ Mesh complications, such as exposure, extrusion, and perforation are uncommon, estimated to occur in approximately $2 \%$ of cases..$^{3,5}$ Urethral perforations are particularly rare with an incidence of $0-0.8 \% .^{3,5}$ The American College of Obstetricians and Gynecologists and American Urogynecologic Society recommend urgent surgical excision for the management of mesh complications. ${ }^{6}$ Surgical options include endoscopic urethral approach, a transvaginal approach, or a staged approach with endoscopic mesh lysis followed by transvaginal excision. For extensive urethral injuries, reconstructive procedures such as a urethroplasty or urethral-vaginal fistula repair may be needed.

Here, we present a case of urethral mesh perforation where surgical excision was delayed due to concurrent presentation of vulvar cancer. This delay resulted in the mesh eroding through the urethra completely and resolution of the patient's irritative voiding symptoms without invasive mesh excision.

\section{Case report}

The patient is a 66-year-old female with obstetric history including one pregnancy with vaginal delivery. Her medical history was significant for a body mass index of 38 and vulvar lichen sclerosis. She was initially seen at another centre sixteen years ago for SUI and underwent a retropubic mid-urethral sling (MUS) procedure. The initial procedure itself was uncomplicated, but provided limited improvement in incontinence. She was seen 5 years later for worsening incontinence and was considered for a pessary but did not undergo further follow up or investigation. 
Sixteen years after her initial MUS she represented with dysuria, frequency, urgency, nocturia, and worsening incontinence requiring multiple pads daily. Her Questionnaire for Urinary Incontinence Diagnosis score was 14/15 for stress incontinence and 9/15 for urge incontinence. ${ }^{7}$ Urinalysis and culture was unremarkable. Her exam showed a grade 2 cystocele, grade 1 uterine prolapse, and grade 2 rectocele. There was also an incidental finding of multiple erythematous periclitoral and labial dermal lesions. Urodynamics were consistent with SUI. During cystoscopy, there was difficulty inserting the 16-French cystoscope. Further examination revealed obstruction due to mesh perforation with the sling traversing the urethral lumen and a calculus encrusted on the exposed mesh. The urothelium anterior and posterior to the mesh appeared healthy and there was no evidence of fistulization. At this time, she was referred to us for excision of the eroded mesh and concurrently referred to gynecologic oncology for biopsy of the vulvar lesions.

The vulvar lesions were found to be multifocal invasive squamous cell carcinoma and high-grade vulvar intraepithelial neoplasia. Radical vulvectomy and bilateral inguinal lymphadenectomy was recommended. A concurrent procedure for the mesh perforation was arranged. After discussing treatment options, the patient opted for endoscopic approach at the time of her vulvectomy and to defer further extensive management until after her recovery.

The vulvectomy and lymphadenectomy was uncomplicated. Cystoscopy showed the mesh had migrated anteriorly and was now located in the ventral wall of the urethra with the calculus on its surface. A small curved hemostat was passed alongside the scope to break and remove the calculus. The underlying mesh remained embedded in the anterior urethral wall without obvious insertion points. A suprameatal approach for excision would have been possible, however, this was deferred due to the proximity to the vulvar lesions and the patient's preference for conservative measures. The vulvar pathology showed high grade invasive squamous cell carcinoma with negative margins and lymph nodes. Analysis showed calcium carbonate/calcium phosphate with magnesium ammonium phosphate calculus.

She returned for cystoscopy five months after the previous surgery. The cystoscope entered the bladder without obstruction. The urethra showed no evidence of calculi or mesh perforation. There was limited erythema at the anterior surface of the urethra, where the mesh had been seen previously. It appeared that the mesh had traversed the entire urethral lumen. The cystoscopy was unremarkable, with resolution of the previously seen mesh. The patient reported improvement in her irritative symptoms, including resolution of her dysuria and SUI, and had a negative cough stress test. She had persistent mild incontinence that was well-tolerated with a security pad and continued to improve from baseline. Given her cystoscopic and clinical improvement, she did not undergo further intervention. 


\section{Discussion}

Mesh complications in vaginal surgery have been controversial in the last decade. Despite the recommendations against mesh for prolapse, the use of mesh for the treatment of SUI is supported by strong evidence and by the American Urological Association and the Canadian Urological Association. ${ }^{8}$, While it has been demonstrated to be effective, it comes with its own complication rates, including a $0.03 \%$ to $0.8 \%$ risk of urethral perforation for suburethral slings. ${ }^{3}$

To our knowledge, there is minimal literature describing the natural history or conservative treatment of urethral mesh perforation. Although detailed guideline recommendations are limited due to the paucity of data for this presentation, the information available all recommend either endoscopic or transvaginal excision of the eroded mesh. ${ }^{6}$ However, due to the patient's concurrent diagnosis of vulvar malignancy, the planned intervention was delayed. As a result, we witnessed the natural history of urethral mesh perforation which ultimately did not require excision.

This is the first report of resolution of urethral mesh perforation we found in literature. Although the patient did require management of the calculus that had formed on the mesh, after it was removed the mesh continued to erode through the urethral lumen and completely traversed the urethra. It is possible that the anterior erosion was halted by calculus formation, and removal of the calculus allowed further anterior migration of the mesh out of the urethra and uninhibited repair of the urothelium. The mechanism through which her incontinence improved after the mesh erosion completed is not clear. We hypothesize that the exposed mesh and associated calculus prevented complete coaptation of the urethra, which contributed to her SUI. Once the erosion resolved, her irritative symptoms improved significantly and the residual incontinence was not bothersome.

While this has not previously been reported in literature, there is physiologic plausibility of resolution of urethral mesh perforation. Typical management of mesh perforation involves excision of the protruding mesh but this often leaves mesh remnants in the urethral wall. In most cases, the urothelium heals over these residual stumps with time. ${ }^{10}$ In this case, it appeared that mesh had eroded to the extent that the urethra healed over the remaining defect.

It is important to note that the generalizability of this case is limited. Particular attention should be paid to risk factors for impaired healing of the urethra. Risk factors for poor wound healing include older age, obesity, cigarette use, diabetes mellitus, poor nutrition, chronic steroid use, immunosuppression, radiation, and chemotherapy. ${ }^{11,12}$ It is important to note that a complete mesh erosion, with mesh ventral to the urethra, should be considered in complex cases.

\section{Conclusions}

This was an unusual case where the standard of care was delayed due to concurrent medical issues. Guidelines and standard practice remain consistent on the need for excision of a urethral mesh perforation. However, our case demonstrates that resolution of mesh complications with 
minimal intervention is possible, and that greater consideration of conservative management may be warranted.

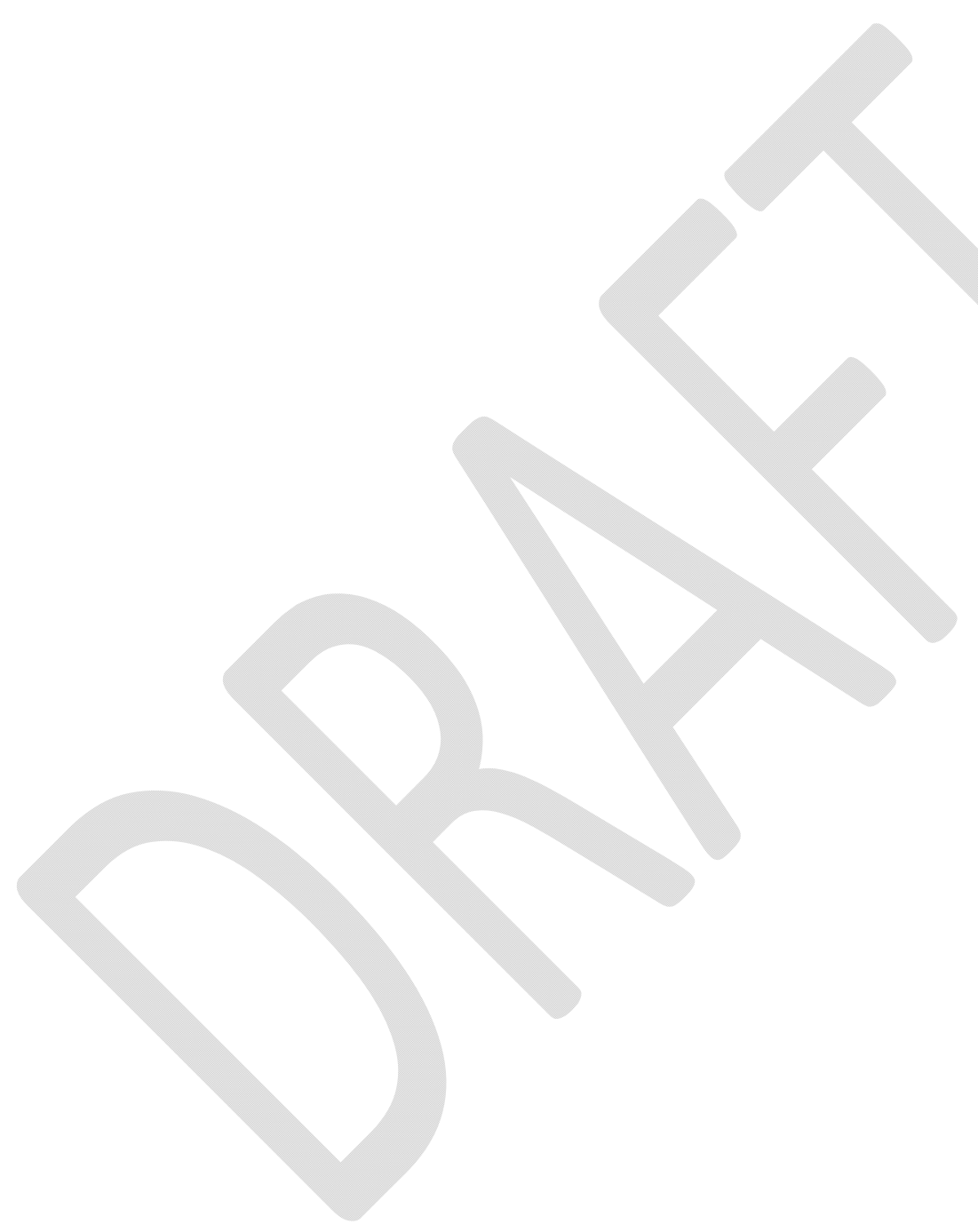




\section{References}

1. Hampel C, Artibani W, Espuña Pons M et al: Understanding the burden of stress urinary incontinence in Europe: a qualitative review of the literature. Eur Urol 2004;46:15.

2. Serati M, Braga A, Athanasiou S, et al. Tension-free vaginal tape-obturator for treatment of pure urodynamic stress urinary incontinence: efficacy and adverse effects at 10-year follow-up. Eur Urol 2017;71:674-9.

3. Gomes CM, Carvalho FL, Bellucci CH, et al. Update on complications of synthetic suburethral slings. Int Braz J Urol 2017 Oct;43:822-34.

4. Ford AA, Rogerson L, Cody JD, et al. Mid-urethral sling operations for stress urinary incontinence in women. Cochrane Database Syst Rev 2017(7).

5. Colhoun A, Rapp DE. Long-term outcomes after repair of transurethral perforation of midurethral sling. Female Pelvic Med Reconstr Surg 2016;22:272-5.

6. American College of Obstetricians and Gynecologists, American Urogynecologic Society. Management of mesh and graft complications in gynecologic surgery. Committee Opinion No. 694. Obstet Gynecol 2017;129:e102-8.

7. Bradley CS, Rahn DD, Nygaard IE, et al. The questionnaire for urinary incontinence diagnosis (QUID): Validity and responsiveness to change in women undergoing nonsurgical therapies for treatment of stress predominant urinary incontinence. Neurourol Urodyn 2010;29:727-34.

8. American Urological Association. AUA Position Statement on the use of vaginal mesh for the surgical treatment of stress urinary incontinence [updated 2019 May; cited 2020 Jan 9]. https://www.auanet.org/guidelines/use-of-vaginal-mesh-for-the-surgicaltreatment-of-stress-urinary-incontinence. Accessed Jan 9, 2020

9. Welk B, Carlson KV, Baverstock RJ, et al. Canadian Urological Association position statement on the use of transvaginal mesh. Can Urol Assoc J 2017;11:S105.

10. Lee $\mathrm{CH}, \mathrm{Ku} \mathrm{JY}$, Lee $\mathrm{K}$, et al. Clinical application of a transurethral holmium laser excision of exposed polypropylene mesh at lower urinary tract: single surgeon experience with long-term follow-up. Female Pelvic Med Reconstr Surg 2018;24:26-31.

11. Buggy D. Can anaesthetic management influence surgical-wound healing?. Lancet 2000;356:355-7.

12. Payne WG, Naidu DK, Wheeler CK, et al. Wound healing in patients with cancer. Eplasty 2008;8. 